\title{
The Proteasome Activator PA200 Regulates Tumor Cell Responsiveness to Glutamine and Resistance to lonizing Radiation
}

\author{
Jennifer Blickwedehl ${ }^{1}$, Scott Olejniczak ${ }^{3}$, Ryan Cummings ${ }^{1}$, Nilofar Sarvaiya ${ }^{1}$, Ana \\ Mantilla ${ }^{1}$, Asher Chanan-Khan ${ }^{2}$, Tej K. Pandita ${ }^{4}$, Marion Schmidt ${ }^{5}$, Craig B. Thompson ${ }^{3}$, \\ and Naveen Bangia ${ }^{1}$ \\ ${ }^{1}$ Department of Immunology, Roswell Park Cancer Institute, Buffalo \\ ${ }^{2}$ Department of Medicine, Roswell Park Cancer Institute, Buffalo \\ ${ }^{3}$ Memorial Sloan-Kettering Cancer Center, New York \\ ${ }^{4}$ Department of Radiation Oncology University of Texas Southwestern, Dallas, Texas \\ ${ }^{5}$ Department of Biochemistry, Albert Einstein College of Medicine Bronx, New York
}

\begin{abstract}
The cellular response to ionizing radiation (IR) involves a variety of mechanisms to repair damage and maintain cell survival. We previously reported that the proteasome activator PA200 promotes long-term cell survival after IR exposure. The molecular function of PA200 is to enhance proteasome-mediated cleavage after glutamate; however, it is not known how this molecular function promotes survival after IR exposure. Here, we report that upon IR exposure, cellular demand for exogenous glutamine is increased. Cells containing PA200 are capable of surviving this IR-induced glutamine demand, whereas PA200-deficient cells show impaired long-term survival. Additional glutamine supplementation reverses the radiosensitivity of PA200-knockdown cells suggesting impaired glutamine homeostasis in these cells. Indeed, PA200-knockdown cells are unable to maintain intracellular glutamine levels. Furthermore, when extracellular glutamine is limiting, cells that contain PA200 respond by slowing growth, but PA200-knockdown cells and cells in which post-glutamyl proteasome activity is inhibited are nonresponsive and continue rapid growth. This cellular unresponsiveness to nutrient depletion is also reflected at the level of the mTOR substrate ribosomal S6 kinase (S6K). Thus, inability to restrict growth causes PA200deficient cells to continue growing and eventually die due to lack of available glutamine. Together, these data indicate an important role for PA200 and post-glutamyl proteasome activity in maintaining glutamine homeostasis, which appears to be especially important for long-term survival of tumor cells after radiation exposure.
\end{abstract}

Corresponding Author: Naveen Bangia, The Binding Site Inc., 5889 Oberlin Drive, Suite 101, San Diego, CA 92121. naveen.bangia@gmail.com.

Current address for N. Bangia: The Binding Site Inc., 5889 Oberlin Drive, Suite 101, San Diego, CA 92121

Note: Supplementary data for this article are available at Molecular Cancer Research Online (http://mcr.aacrjournals.org/).

Disclosure of Potential Conflicts of Interest

No potential conflicts of interests were disclosed by the authors. 


\section{Introduction}

The decision of tumor cells to grow or not depends on multiple signals including those from available nutrients, extracellular growth factors, damage, and stress. Stressors such as ionizing radiation (IR) slow cell growth due to cellular responses that attempt to repair DNA damage before replication (reviewed in ref. 1). IR induces reactive oxygen species and double-strand DNA breaks, both of which are sensed by elaborate detection and signaling cascades that lead to the arrest of cell cycle and induction of DNA repair proteins (reviewed in refs. 1-3). Continued growth through cell-cycle checkpoints (i.e., inability to arrest cell cycle) in response to DNA damage is associated with genomic instability and diminished long-term cell survival $(4,5)$. Thus, defects in proteins involved in sensing or repairing DNA damage are known to impair survival as measured by clonogenic survival assays $(4,6,7)$. We have recently reported that depletion of the proteasome activator, PA200, from tumor cells impairs their clonogenic survival after radiation exposure (8). However, the mechanism by which PA200 promotes survival has not yet been reported.

The proteasome activator, PA200, is a heat/armadillo repeat protein that binds to the ends of core (or 20S) proteasomes $(9,10)$ and responds to $\operatorname{IR}(8,11)$. Core proteasomes contain 3 active sites (chymotryptic, tryptic, and post-glutamyl) to degrade proteins. The caps (i.e., PA200 cap, 19S cap) that bind the ends of the barrel shaped core proteasome regulate the entry of substrates (reviewed in ref. 12). PA200 association enhances proteasome-mediated cleavage of peptides after the amino acid glutamate (postglutamyl activity; ref. 11). We previously showed that IR enhanced cellular levels of PA200-19S hybrid proteasomes, which are composed of the core proteasome capped on one end by PA200 and a 19S cap on the other end (8). Furthermore, PA200 diminution or specific inhibition of post-glutamyl proteasome activity impaired cell survival after IR exposure (8). Thus, PA200 and hybrid proteasomes, through post-glutamyl proteasome activity, promote survival after radiation exposure. Because glutamate is a precursor for intracellular glutamine generation, we investigated the relationship between radiation and glutamine homeostasis and how PA200 affects resistance to radiation through glutamine homeostasis.

Glutamine is a particularly important amino acid as a precursor for nucleotide, protein, and lipid biosynthesis as well as promoting mTOR activity (13-16). Glutamine is also rapidly converted into glutamate, which can enter the tricarboxylic acid (TCA) cycle for ATP production $(14,17)$. The central importance of glutamine and glutamate in energy and biosynthetic pathways may explain why tumor cells consume large quantities of glutamine for growth and proliferation (15, 17-19). However, the importance of glutamine homeostasis after exposure of cells to stressors such as IR is not understood. Here, we investigate how PA200 modulates radiation sensitivity through glutamine metabolism.

\section{Materials and Methods}

\section{Cell lines, antibodies, and irradiation}

HeLa cervical carcinoma and B16.F10 murine melanoma cell lines were grown in RPMI supplemented with $2 \mathrm{mmol} / \mathrm{L}$ (final) glutamine and 10\% bovine calf serum. BJ human fibroblasts were cultured in Dulbecco's Modified Eagle's Medium (DMEM) supplemented 
with $2 \mathrm{mmol} / \mathrm{L}$ glutamine (final) and $10 \%$ bovine calf serum. Rabbit anti-sera recognizing PA200 has been previously described (20). All other antibodies are detailed in Supplementary Methods. Transfection was conducted using Amaxa Nucleofector (Lonza) as detailed in Supplementary Methods. Radiation exposure was conducted using Philips Mark 1 Cesium irradiator at a dose rate of $0.472 \mathrm{~Gy} / \mathrm{min}$ as detailed in Supplementary Methods. Different dose rates of irradiation were used because of change of irradiation equipment at Roswell Park Cancer Institute (RPCI; Buffalo, NY) during the course of these studies.

\section{Nutrient depletion}

Immediately after transfection, cells were plated at the indicated densities in 6-well dishes $\left(14.4 \times 10^{4}\right.$ cells $\left./ \mathrm{cm}^{2}\right)$ in $3 \mathrm{~mL}$ of media. For nutrient-replete conditions, media were replaced daily. Seventy-two hours after transfection, cells were lysed directly in the dish with $1 \%$ Triton X-100, $0.5 \mathrm{mmol} / \mathrm{L}$ phenylmethylsulfonylfluoride (PMSF), $5 \mathrm{~mol} / \mathrm{L} N$ ethylmaleimide (NEM), $1 \mathrm{mmol} / \mathrm{L} \mathrm{NaF}, 5 \mathrm{mmol} / \mathrm{L} \beta$-glycerophosphate, in TBS.

\section{Results}

Exposure of cells to IR enhanced consumption of glutamine from the media compared with nonirradiated cells (Fig. 1A). Because PA200-knockdown cells show impaired survival after IR exposure (8), we hypothesized that it may be due to the inability to survive and grow from this IR-induced glutamine demand. Therefore, we tested whether excess extracellular glutamine could reverse the radiosensitivity that we previously observed in PA200knockdown cells. We compared clonogenic survival (which is a result of both survival and proliferation) of HeLa cells with and without knockdown of PA200 (Fig. 1B), in standard growth media (containing $2 \mathrm{mmol} / \mathrm{L}$ glutamine) versus media that had been supplemented with extra glutamine midway (day 7) through the 13-day clonogenic assay. Strikingly, extra glutamine addition alone enhanced survival of PA200-knockdown cells, but not control cells, after radiation exposure (Fig. 1C and D). In the presence of excess glutamine added 7 days after irradiation, PA200-knockdown cells no longer show radiosensitivity but appear radioresistant compared with control siRNA-transfected cells (Fig. 1D). Together, these data suggest that radiation induces an increased glutamine demand in cells to an extent that glutamine in the media become limiting in a long-term 13-day clonogenic survival assay (for PA200-knockdown cells). Consistent with this idea, when colonies were examined on day 7 (rather than day 13), PA200-knockdown cells do not show radiosensitivity but rather show enhanced colony formation compared with control siRNA-transfected cells (Fig. 1E). Thus, 7 days after irradiation, PA200-knockdown cells grow better than control siRNA-transfected cells.

One possibility for why PA200-depleted cells show enhanced growth when measured shortly after radiation (day 7) and why glutamine supplementation restores survival at day 13 after radiation is that PA200-knockdown cells might take up more glutamine from the media, thereby exhausting it sooner than cells that contain PA200. Consistent with this idea, the cell culture media of PA200-knockdown cells are depleted of glutamine more rapidly than control siRNA-transfected cells (Fig. 2A, compare black bar to white bar on day 2 after transfection). However, this increased glutamine uptake did not result in increased growth in 
typical growth media (which contains $2 \mathrm{mmol} / \mathrm{L}$ glutamine). Because typical growth media contain excess $(2 \mathrm{mmol} / \mathrm{L})$ glutamine for proliferation (21), we hypothesized that when glutamine conditions were low, the increased glutamine uptake by PA200-knockdown cells might promote enhanced growth. In low-glutamine concentrations, colony formation (without radiation exposure) of PA200-depleted cells is greater than control siRNAtransfected cells (Fig. 2B). Cell growth is saturated at $0.2 \mathrm{mmol} / \mathrm{L}$ or more, where colony formation (on day 13) is comparable between control siRNA-transfected and PA200knockdown cells (Fig. 2B). Similarly, enhanced growth of PA200-knockdown cells compared with control siRNA-transfected cells in low $(0.05 \mathrm{mmol} / \mathrm{L})$-glutamine conditions is apparent in short-term growth assays (Fig. 2C). Because PA200 enhances post-glutamyl proteasome activity, we tested whether specific inhibition of post-glutamyl proteasome activity caused the same phenotype of enhanced growth in low $(0.05 \mathrm{mmol} / \mathrm{L})$ glutamine. Like PA200 inhibition, specific inhibition of post-glutamyl proteasome activity using azNC-001 (Supplementary Fig. S1A; ref. 22) enhanced short-term growth in low (0.05 $\mathrm{mmol} / \mathrm{L}$ )-glutamine conditions but not in higher $(0.2 \mathrm{mmol} / \mathrm{L})$-glutamine conditions (Fig. 2D and E). Therefore, PA200-knockdown cells and cells in which post-glutamyl activity is specifically inhibited show enhanced growth after short-term exposure to low-glutamine conditions (even in the absence of irradiation). The enhanced growth of PA200-knockdown cells in the short-term is associated with diminished long-term growth (Supplementary Fig. S1B), consistent with the idea that deficiency in PA200 leads to enhanced growth in nutrient-deprived settings, but eventual death due to inability to restrict growth.

We hypothesized that PA200-knockdown cells grow better (in the short-term) in lowglutamine conditions because control cells limit their growth in response to glutamine limitation. From this perspective, PA200-knockdown cells do not appropriately restrict their growth in low-glutamine conditions. Therefore, we tested whether PA200-knockdown cells could restrict their growth in response to nutrient depletion that occurs over time in culture. We compared a nutrient-depleted setting with a nutrient-replete setting (i.e., complete nutrient replacement). As expected, control siRNA-transfected cells whose nutrients were replaced daily showed greater cell recovery than those without nutrient replacement (Fig. 3A, black bars). PA200-knockdown cells continued with elevated cell growth even when nutrients were depleted (Fig. 3A, white bars). Restriction of growth in response to nutrient or amino acid deprivation is determined by mTOR (23), which integrates signals from growth factors, nutrient availability, and stressors to determine whether a cell grows or not (24). A direct target of mTOR kinase activity is ribosomal S6 kinase (S6K; refs. 25, 26). Thus, as a second measure to investigate growth restriction in response to nutrient limitation, we used mTOR-mediated phosphorylation of S6K (p-S6K) as a readout. Control siRNAtransfected cells show low levels of p-S6K in nutrient-depleted settings compared with high levels in nutrient-replete conditions suggestive of responsiveness of mTOR activity to nutrient depletion and stimulation (Fig. 3B, lane 2 vs. 1). When nutrients were depleted, PA200-knockdown cells maintain high levels of p-S6K than in control cells (Fig. 3B, lane 3 vs. 1). Furthermore, nutrient replacement does not increase p-S6K in PA200-knockdown cells (Fig. 3B, lane 3 vs. 4). Similar experiments carried out at lower cell density $\left(3.33 \times 10^{4}\right.$ cells $/ \mathrm{cm}^{2}$ ) contained sufficient nutrients such that $\mathrm{p}-\mathrm{S} 6 \mathrm{~K}$ remained high and unchanged, and cell yield was unchanged by media replacement (see Supplementary Fig. S2A). Thus, the 
effects of PA200 deficiency are observed most dramatically during nutrient deprivation and growth restriction that occurs at higher cell densities. These data show that when PA200 is depleted, $\mathrm{p}$-S6K is nonresponsive to nutrient stress that occurs in nutrient-deprived cultures over time.

If only nutrient-replete conditions are compared, control siRNA-transfected cells are stimulated by nutrients and show higher levels of p-S6K than PA200 siRNA-transfected cells (Fig. 3B, compare lane 2 with 4, respectively). This phenotype of higher p-S6K in control siRNA-transfected cells than in PA200-knockdown cells (in nutrient-replete conditions) is reproduced in human (HeLa, BJ fibroblasts) and mouse (B16.F10 melanoma) cell lines (Fig. 3C). Consistent with blunted responsiveness to nutrients in PA200knockdown cells, S6K phosphorylation after serum stimulation is also impaired (Fig. 3D).

Because proteasomes degrade proteins, we considered the possibility that lack of nutrient responsiveness in PA200-knockdown cells could be due to degradation or altered levels of components of the mTOR complex. Our results do not support this idea because mTOR, Raptor, and Rictor are all appreciably expressed in both PA200-knockdown and control cells (Fig. 3E). Furthermore, phosphorylated (active) forms of mTOR representing mTORC1 and mTORC2 seem to be present at high levels both in control and PA200 siRNA-transfected cells (Fig. 3E). Because mTOR activity directly correlates with cell size (27), we compared the size of cells with and without PA200 and found no difference (Fig. 3F). Rapamycin, a specific mTOR inhibitor, diminishes cell size of both PA200 and control siRNA-transfected cells, showing that mTOR is likely active and can be inhibited by rapamycin in PA200knockdown cells (Fig. 3F). Furthermore, rapamycin also inhibited the p-S6K levels in both control and PA200-knockdown cells (Supplementary Fig. S2B). These data show that mTOR components are apparently not degraded in PA200-depleted cells. mTOR function per se is likely intact in PA200-knockdown cells, however, it is the responsiveness of mTOR targets that is altered.

Because PA200 alters pS6K responsiveness and mTOR activity has previously been shown to be regulated in response to DNA damage $(28,29)$, we examined the effects of PA200 on IR-induced changes in pS6K. In the presence of PA200, IR exposure leads to a dramatic decrease in p-S6K between 1 and 4 hours postirradiation (Figs. 3G and $\mathrm{H}$ and 4, and 24 hours after IR). However, p-S6K levels in PA200-depleted cells are not diminished 1 and 4 hours postirradiation, rather they are increased at 4 and 24 hours postirradiation (Fig. 3G and $\mathrm{H})$. These data suggest that IR exposure restrains growth (i.e., lowers p-S6K levels) of control cells, but not PA200-knockdown cells.

The enhanced growth of PA200-knockdown cells in glutamine-limiting conditions led us to question whether PA200-depleted cells are more dependent on an absolute requirement for extracellular glutamine for their survival than cells containing PA200. Therefore, we tested cell growth $\left(3 \times 10^{4}\right.$ cells $\left./ \mathrm{cm}^{2}\right)$ in media completely devoid of both glutamine and its precursor glutamate (Fig. 4A). In this case, PA200-depleted cells show impaired growth after extended periods without glutamine and glutamate (Fig. 4A). The defect in cell growth of PA200-knockdown cells in glutamine- and glutamate-deficient media was specific as removal of glucose or serum led to decreases in growth that were identical between PA200- 
knockdown and control cells (Fig. 4A). Thus, PA200-knockdown cells show impaired growth in the complete absence of glutamine and glutamate, suggesting that these cells have impaired glutamine homeostasis and are therefore more dependent on extracellular glutamine and/or glutamate.

The observation of impaired growth of PA200-depleted cells in the complete absence of extracellular glutamine and glutamate suggests that PA200, which promotes post-glutamyl cleavage by proteasomes, may be required to replenish cells with glutamate and/or glutamine under complete starvation conditions. Consistent with this idea, PA200-depleted cells show diminished intracellular free glutamine levels compared with cells with PA200 (Fig. 4B). This decrease in glutamine is specific as other amino acids such as valine and arginine remained at wild-type levels in PA200-knockdown cells. Although PA200knockdown cells seem to consume more glutamine from the extracellular media (Fig. 2A), they show diminished intracellular free glutamine. Upon uptake of glutamine into cells, it is rapidly converted to glutamate, of which some is released back into the media. Interestingly, PA200-depleted cells show diminished glutamate release into the media (Fig. 4C, compare black bar with white bar). This diminution can be reversed by introduction of PA200 cDNA that is insensitive to the siRNA (Fig. 4C compare white bar with gray bar). In summary, loss of PA200 results in decreased intracellular glutamine levels and consequently less glutamate is excreted into the media. These data argue that PA200 is required for maintenance of intracellular glutamine homeostasis.

\section{Discussion}

Here, we show that IR exposure enhances glutamine consumption and that the radiation sensitivity of PA200-deficient cells is directly related to impaired glutamine homeostasis after IR exposure. Exogenous glutamine supplementation restores resistance of PA200knockdown cells to IR exposure. PA200-knockdown cells seem to be more dependent on exogenous glutamine to compensate for impaired maintenance of intracellular glutamine levels. We further show that these cells do not restrict growth in conditions of low glutamine and continue attempted growth, which likely leads to their demise in a long-term clonogenic survival assay. The inability to restrict cell growth in nutrient-deprived settings is associated with dysregulated mTOR responsiveness.

Our observation that glutamine is depleted from the supernatant after IR treatment is consistent with microarray data, indicating that the glutamine importers SLC7A6 and SLC7A5 are increased after radiation $(30,31)$. Furthermore, the glutamine exporter SLC7A7 is diminished after radiation $(30,31)$. Together, these data suggest that IR induces a greater demand for glutamine, which is compensated by increased uptake of extracellular glutamine.

The demand for glutamine after IR exposure appears to require PA200 because PA200knockdown HeLa cells exhibit increased radiosensitivity, which is reversed by supplementing cells with additional exogenous glutamine levels. Therefore, glutamine may be a limiting constituent after radiation treatment especially for cells lacking PA200, as these cells do not survive in long-term clonogenic survival assay. Our ability to identify a survival defect in PA200-knockdown cells was only possible because we used a long-term 
clonogenic assay without feeding (10-14 days). In vitro clonogenic survival of tumor cells after radiation has been reported 7 days (32) after radiation or 10 to 14 days after radiation $(8,33)$. Because very few cells are plated, they are not expected to deplete the nutrients available in the media and thus media are often not replaced or cultures are not refed during the 7- or 10- to 14-day clonogenic survival assays (in some studies, media are replaced). Because we show that PA200-knockdown cells form more colonies than control siRNAtransfected cells on day 7, but fewer colonies than control cells on day 13 after radiation, we suggest that proteins that affect glutamine homeostasis or responsiveness to glutamine can impact long-term clonogenic survival as determined on day 10 to 14 (without feeding).

The increased growth in low-glutamine conditions in the absence of PA200 might indicate an adaptation caused by limiting intracellular glutamine levels. These cells also continue to grow and maintain high mTOR activity (p-S6K levels), whereas control cells slow cell growth and dampen mTOR activity in nutrient-deprived settings. Furthermore, we show that IR exposure elicits a transient decrease in p-S6K in control cells that is not apparent in PA200-knockdown cells. Two previous studies have examined the effects of DNA-damaging agents on mTOR and the mTOR substrate 4EBP1. One report suggests enhancement of mTOR activity after IR exposure (28), whereas another suggests inhibition of mTOR activity (by measuring 4EBP phosphorylation) after etoposide-induced DNA damage (29). Thus, the effect of DNA damage on mTOR appears to be context-dependent. However, a recent report shows that DNA damage-mediated inhibition of S6K phosphorylation requires ATM, liver kinase B1 (LKB1), and 5' -adenosine monophosphate-activated protein kinase (AMPK; ref. 34). How PA200 deficiency affects this pathway of regulation of S6K remains to be elucidated.

Why and how is mTOR potentially dysregulated in PA200-knockdown cells? Nutrient sensing by mTOR is known to be regulated by amino acid availability. mTOR integrates signals from amino acid availability, insulin, growth factors, and stressors (35). A previous study suggests that proteasome activity is required for responsiveness of mTOR and thus S6K to insulin (36). These authors suggested that proteasomes contribute to the homeostasis of intracellular amino acid pools and are therefore necessary to provide the "building block" or "amino acid" signal for mTOR activation. Our observation that PA200-knockdown cells show growth impairment in media completely devoid of glutamine (and glutamate) suggests that PA200 and proteasome catabolic activity may be important to recover intracellular glutamine or glutamate when these amino acids are not available in the extracellular space. Because we have previously shown increased cellular abundance of PA200-19S hybrid proteasomes after radiation exposure (8), we suggest that specialized proteasomes such as PA200-19S hybrid proteasomes, which shift the peptidase specificity of the proteasome, may be required for the generation of free amino acids such as glutamate or glutamine which regulate mTOR signaling. In the absence of PA200 or post-glutamyl proteasome activity to provide intracellular free glutamine, there may be still unknown compensatory mechanisms that increase glutamine uptake and promote sustained mTOR activity, but which are unresponsive to nutrient depletion or stimulation.

Tumor cell survival from radiation appears to be a complex phenomenon that encompasses sensing, repairing, and recovering from cell-cycle checkpoints after DNA damage. In 
addition to these effects, we reveal here that IR exposure leads to an increased demand for glutamine. Furthermore, we show a specific role for PA200 and glutamine homeostasis in long-term tumor cell survival after radiation exposure. How PA200 impacts tumor resistance to radiation in vivo and the biology of PA200 and glutamine homeostasis after in vivo radiation remains to be explored.

\section{Supplementary Material}

Refer to Web version on PubMed Central for supplementary material.

\section{Acknowledgments}

The authors thank Alexei Kisselev (Dartmouth University, Hanover, NH) for the kind gift of the post-glutamylspecific inhibitor az-NC-001. The authors also thank Amy Stewart, Jennifer Pearce, and Melanie Farrauto for assistance with irradiations.

\section{Grant Support}

This work was supported by Investigator Start-Up Funds (to N. Bangia), NIH grant GM-084228 (to M. Schmidt), CA129537 (to T.K. Pandita), CA154320 (to T.K. Pandita), and a National Cancer Institute Cancer Center Support Grant (CA16056) to RPCI.

\section{References}

1. Sancar A, Lindsey-Boltz LA, Unsal-Kaccmaz K, Linn S. Molecular mechanisms of mammalian DNA repair and the DNA damage checkpoints. Annu Rev Biochem. 2004; 73:39-85. [PubMed: 15189136]

2. Gehen SC, Staversky RJ, Bambara RA, Keng PC, O'Reilly MA. hSMG-1 and ATM sequentially and independently regulate the G1 checkpoint during oxidative stress. Oncogene. 2008; 27:4065-4074. [PubMed: 18332866]

3. Rotman G, Shiloh Y. Ataxia-telangiectasia: is ATM a sensor of oxidative damage and stress? Bioessays. 1997; 19:911-917. [PubMed: 9363685]

4. Taylor AM, Harnden DG, Arlett CF, Harcourt SA, Lehmann AR, Stevens S, et al. Ataxia telangiectasia: a human mutation with abnormal radiation sensitivity. Nature. 1975; 258:427-429. [PubMed: 1196376]

5. Zachos G, Rainey MD, Gillespie DA. Chk1-deficient tumour cells are viable but exhibit multiple checkpoint and survival defects. EMBO J. 2003; 22:713-723. [PubMed: 12554671]

6. Sharan SK, Morimatsu M, Albrecht U, Lim DS, Regel E, Dinh C, et al. Embryonic lethality and radiation hypersensitivity mediated by Rad51 in mice lacking Brca2. Nature. 1997; 386:804-810. [PubMed: 9126738]

7. Carney JP, Maser RS, Olivares H, Davis EM, Le Beau M, Yates JR III, et al. The hMre11/hRad50 protein complex and Nijmegen breakage syndrome: linkage of double-strand break repair to the cellular DNA damage response. Cell. 1998; 93:477-486. [PubMed: 9590181]

8. Blickwedehl J, Agarwal M, Seong C, Pandita RK, Melendy T, Sung P, et al. Role for proteasome activator PA200 and postglutamyl proteasome activity in genomic stability. Proc Natl Acad Sci U S A. 2008; 105:16165-16170. [PubMed: 18845680]

9. Schmidt M, Haas W, Crosas B, Santamaria PG, Gygi SP, Walz T, et al. The HEAT repeat protein Blm10 regulates the yeast proteasome by capping the core particle. Nat Struct Mol Biol. 2005; 12:294-303. [PubMed: 15778719]

10. Iwanczyk J, Sadre-Bazzaz K, Ferrell K, Kondrashkina E, Formosa T, Hill CP, et al. Structure of the Blm10-20 S proteasome complex by cryoelectron microscopy. Insights into the mechanism of activation of mature yeast proteasomes. J Mol Biol. 2006; 363:648-659. [PubMed: 16952374]

11. Ustrell V, Hoffman L, Pratt G, Rechsteiner M. PA200, a nuclear proteasome activator involved in DNA repair. EMBO J. 2002; 21:3516-3525. [PubMed: 12093752] 
12. Rechsteiner M, Hill CP. Mobilizing the proteolytic machine: cell biological roles of proteasome activators and inhibitors. Trends Cell Biol. 2005; 15:27-33. [PubMed: 15653075]

13. Kovacevic Z, Jerance D, Brkljac O. The role of glutamine oxidation and the purine nucleotide cycle for adaptation of tumour energetics to the transition from the anaerobic to the aerobic state. Biochem J. 1988; 252:381-386. [PubMed: 3415660]

14. Newsholme P, Procopio J, Lima MM, Pithon-Curi TC, Curi R. Glutamine and glutamate-their central role in cell metabolism and function. Cell Biochem Funct. 2003; 21:1-9. [PubMed: 12579515]

15. DeBerardinis RJ, Mancuso A, Daikhin E, Nissim I, Yudkoff M, Wehrli S, et al. Beyond aerobic glycolysis: transformed cells can engage in glutamine metabolism that exceeds the requirement for protein and nucleotide synthesis. Proc Natl Acad Sci U S A. 2007; 104:19345-19350. [PubMed: 18032601]

16. Nicklin P, Bergman P, Zhang B, Triantafellow E, Wang H, Nyfeler B, et al. Bidirectional transport of amino acids regulates mTOR and autophagy. Cell. 2009; 136:521-534. [PubMed: 19203585]

17. Kovacevic Z, Morris HP. The role of glutamine in the oxidative metabolism of malignant cells. Cancer Res. 1972; 32:326-333. [PubMed: 4400467]

18. Moreadith RW, Lehninger AL. The pathways of glutamate and glutamine oxidation by tumor cell mitochondria. Role of mitochondrial NAD (P)+-dependent malic enzyme. J Biol Chem. 1984; 259:6215-6221. [PubMed: 6144677]

19. Tannock IF, Steele D, Roberts J. Influence of reduced concentration of L-glutamine on growth and viability of cells in monolayer, in spheroids, and in experimental tumours. Br J Cancer. 1986; 54:733-741. [PubMed: 3801270]

20. Blickwedehl J, McEvoy S, Wong I, Kousis P, Clements J, Elliott R, et al. Proteasomes and proteasome activator $200 \mathrm{kDa}$ (PA200) accumulate on chromatin in response to ionizing radiation. Radiat Res. 2007; 167:663-674. [PubMed: 17523843]

21. Baechtel FS, Gregg DE, Prager MD. The influence of glutamine, its decomposition products, and glutaminase on the transformation of human and mouse lymphocytes. Biochim Biophys Acta. 1976; 421:33-43. [PubMed: 1082349]

22. Britton M, Lucas MM, Downey SL, Screen M, Pletnev AA, Verdoes M, et al. Selective inhibitor of proteasome's caspase-like sites sensitizes cells to specific inhibition of chymotrypsin-like sites. Chem Biol. 2009; 16:1278-1289. [PubMed: 20064438]

23. Proud CG. Amino acids and mTOR signalling in anabolic function. Biochem Soc Trans. 2007; 35:1187-1190. [PubMed: 17956308]

24. Yang Q, Guan KL. Expanding mTOR signaling. Cell Res. 2007; 17:666-681. [PubMed: 17680028]

25. Hara K, Yonezawa K, Weng QP, Kozlowski MT, Belham C, Avruch J. Amino acid sufficiency and mTOR regulate p70 S6 kinase and eIF-4E BP1 through a common effector mechanism. J Biol Chem. 1998; 273:14484-14494. [PubMed: 9603962]

26. Isotani S, Hara K, Tokunaga C, Inoue H, Avruch J, Yonezawa K. Immunopurified mammalian target of rapamycin phosphorylates and activates p70 S6 kinase alpha in vitro. J Biol Chem. 1999; 274:34493-34498. [PubMed: 10567431]

27. Fingar DC, Salama S, Tsou C, Harlow E, Blenis J. Mammalian cell size is controlled by mTOR and its downstream targets S6K1 and 4EBP1/eIF4E. Genes Dev. 2002; 16:1472-1487. [PubMed: 12080086]

28. Tirado OM, Mateo-Lozano S, Sanders S, Dettin LE, Notario V. The PCPH oncoprotein antagonizes the proapoptotic role of the mammalian target of rapamycin in the response of normal fibroblasts to ionizing radiation. Cancer Res. 2003; 63:6290-6298. [PubMed: 14559816]

29. Tee AR, Proud CG. DNA-damaging agents cause inactivation of translational regulators linked to mTOR signalling. Oncogene. 2000; 19:3021-3031. [PubMed: 10871854]

30. Tusher VG, Tibshirani R, Chu G. Significance analysis of microarrays applied to the ionizing radiation response. Proc Natl Acad Sci U S A. 2001; 98:5116-5121. [PubMed: 11309499]

31. Rieger KE, Hong WJ, Tusher VG, Tang J, Tibshirani R, Chu G. Toxicity from radiation therapy associated with abnormal transcriptional responses to DNA damage. Proc Natl Acad Sci U S A. 2004; 101:6635-6640. [PubMed: 15096622] 
32. Sanli T, Rashid A, Liu C, Harding S, Bristow RG, Cutz JC, et al. Ionizing radiation activates AMPactivated kinase (AMPK): a target for radio-sensitization of human cancer cells. Int J Radiat Oncol Biol Phys. 2010; 78:221-229. [PubMed: 20615625]

33. Mothersill C, Saroya R, Smith RW, Singh H, Seymour CB. Serum serotonin levels determine the magnitude and type of bystander effects in medium transfer experiments. Radiat Res. 2010; 174:119-123. [PubMed: 20681806]

34. Alexander A, Cai SL, Kim J, Nanez A, Sahin M, MacLean KH, et al. ATM signals to TSC2 in the cytoplasm to regulate mTORC1 in response to ROS. Proc Natl Acad Sci U S A. 2010; 107:41534158. [PubMed: 20160076]

35. Proud CG. mTOR-mediated regulation of translation factors by amino acids. Biochem Biophys Res Commun. 2004; 313:429-436. [PubMed: 14684180]

36. Proud CG. Regulation of mammalian translation factors by nutrients. Eur J Biochem. 2002; 269:5338-5349. [PubMed: 12423332] 

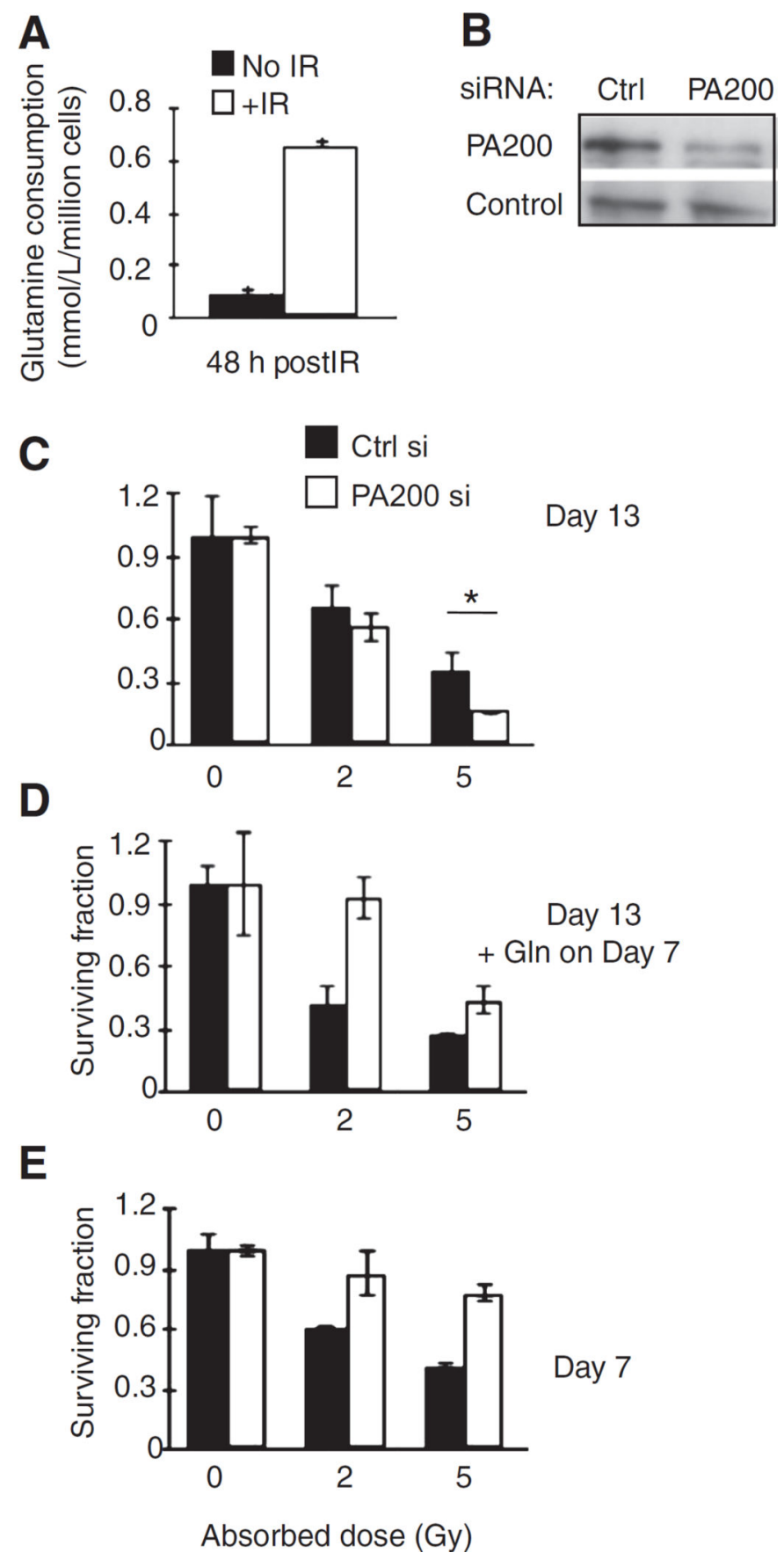

Figure 1.

Glutamine reverses radiosensitivity of PA200-knockdown cells. A, glutamine levels in the supernatant of untransfected HeLa cells after 5-Gy IR were determined using a BioProfile FLEX instrument (Nova Biomedical). Glutamine consumed was calculated as (initial glutamine level before IR-remaining glutamine level 48 hours after IR)/million cells. B-E, HeLa cells transfected with control (Ctrl) or PA200-specific siRNA were incubated for 48 hours and assessed for diminished PA200 protein levels by Western blot analysis. Control (Ctrl) is HSP70 (constitutively expressed in HeLa cells; B). Transfected cells were also 
exposed to 0,2 , or $5 \mathrm{~Gy}$ IR followed by incubation at $37^{\circ} \mathrm{C}, 5 \% \mathrm{CO}_{2}$ for 13 days (C) or 7 days (E). One group of cells (D) was also supplemented on day 7 with $2 \mathrm{mmol} / \mathrm{L}$ glutamine (Gln) and colony formation assessed on day 13 post-IR exposure. A representative figure of at least 3 independent experiments is shown for each panel. 

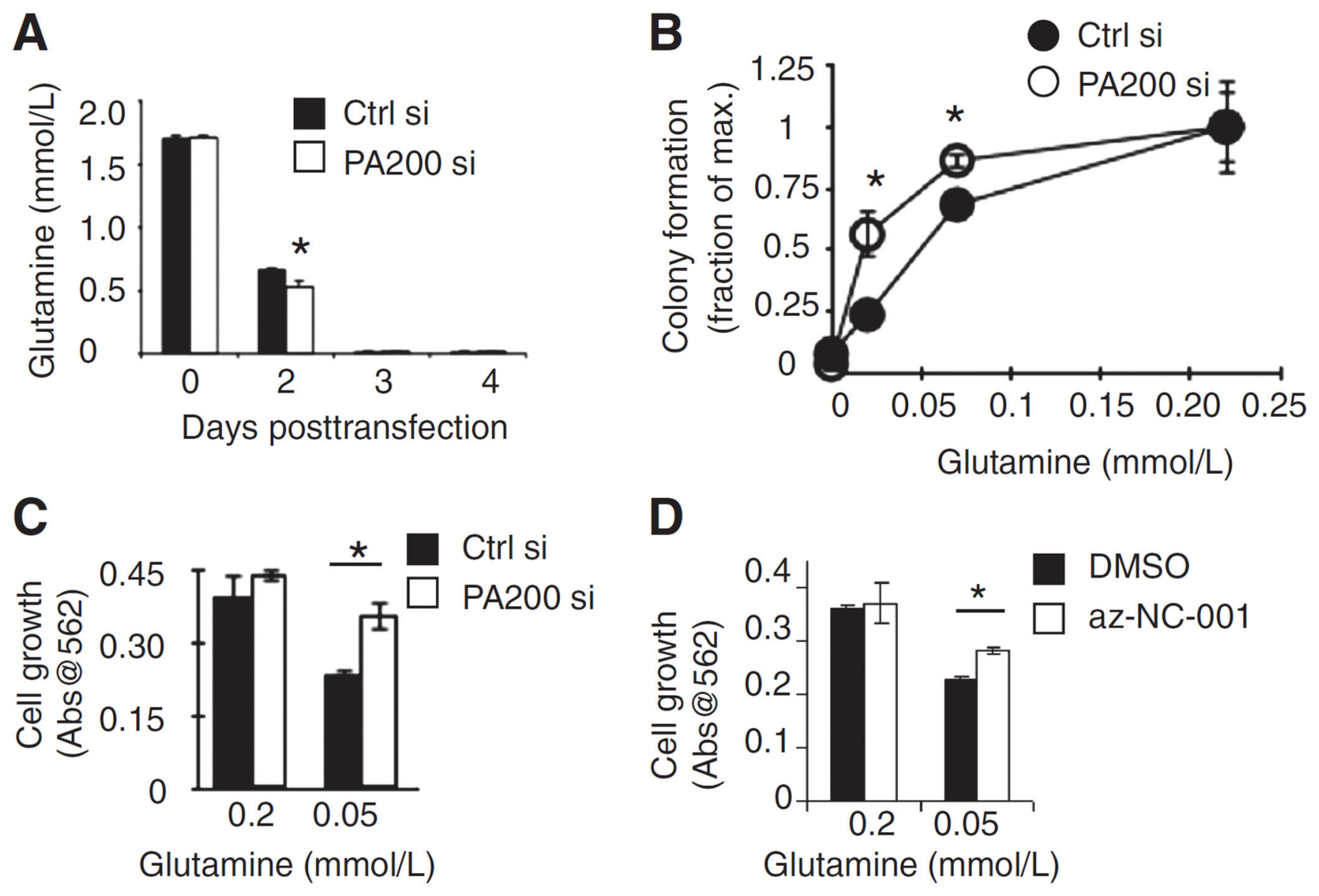

D
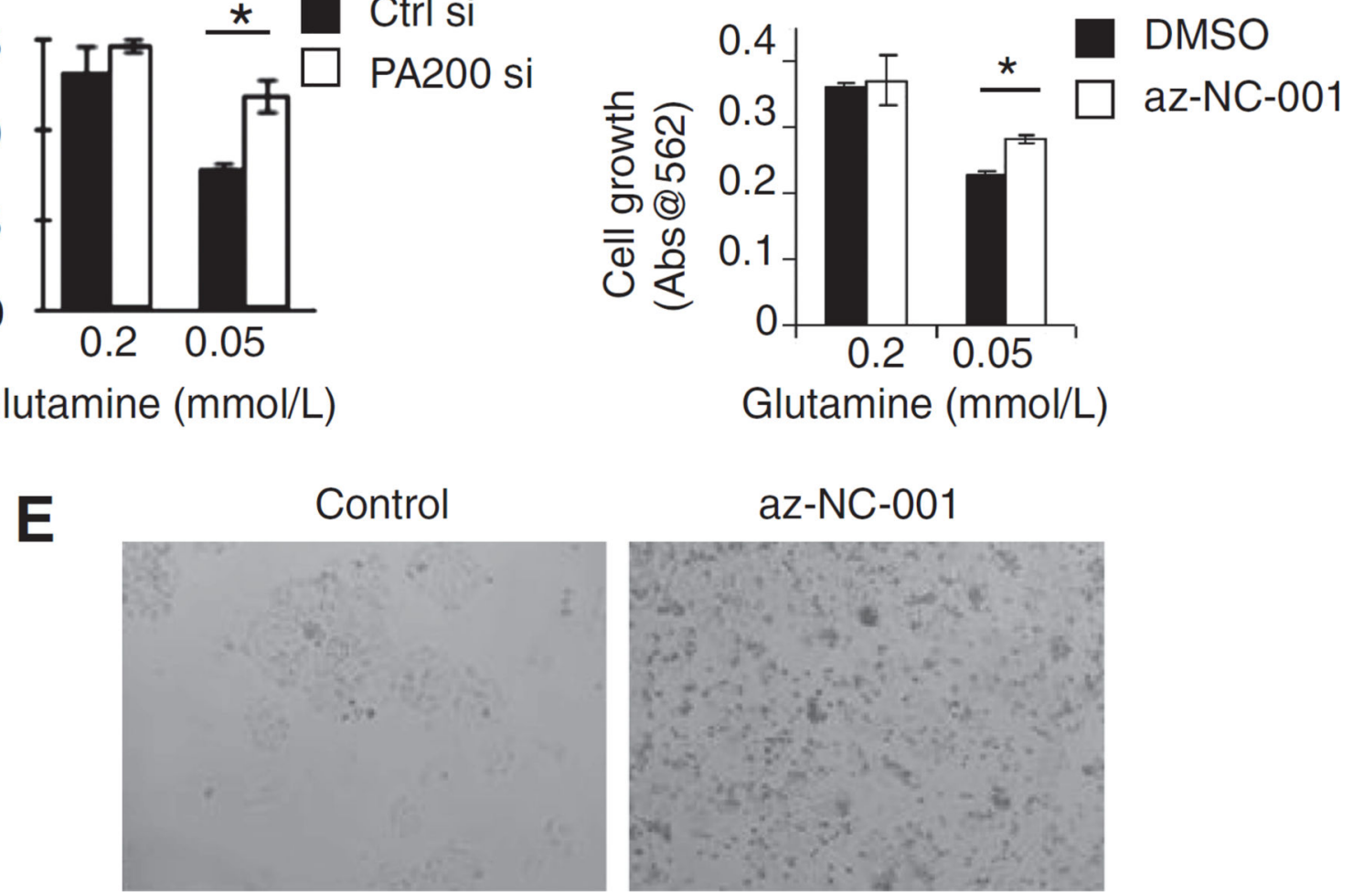

Figure 2.

PA200 affects growth in glutamine-limiting conditions. A, after transfection of HeLa cells and plating in $2 \mathrm{mmol} / \mathrm{L}$ glutamine, the glutamine remaining in the media was measured using a Nova Flex device at each time point. B, clonogenic survival assay using untransfected HeLa cells in media supplemented with indicated concentrations of glutamine. Colony formation was assessed 13 days after plating without media replacement. C, HeLa cells transfected with the PA200-specific siRNA (white bars) or nonspecific siRNA (Ctrl si, black bars) were plated $\left(0.33 \times 10^{4} / \mathrm{cm}^{2}\right)$ in media with 0.05 or $0.2 \mathrm{mmol} / \mathrm{L} \mathrm{L}$-glutamine for 72 hours. Cell growth was assessed using Promega cell proliferation kit (MTS assay). D and E, untransfected HeLa cells were plated at $1 \times 10^{6}$ cells per 10-cm diameter dish in complete 
growth media in the presence of az-NC-001 at $4 \mu \mathrm{mol} / \mathrm{L}$ or dimethyl sulfoxide (DMSO, vehicle) for 48 hours. Cells were harvested and replated at 10,000 cells per well of a 96-well dish in fresh media containing inhibitor az-NC-001 or DMSO for 2 days. Pictures were taken (E) and cell growth was measured by Promega Proliferation Kit (MTS; D). *, $P<$ 0.005. A representative figure of at least 3 independent experiments is shown for each panel. 
A

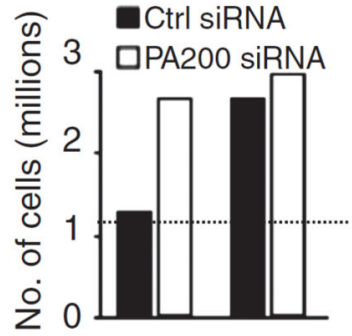

Nutrient: - +

B

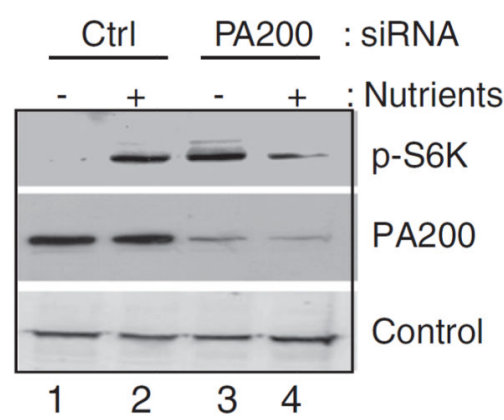

C Nutrient replete

si: $\frac{\mathrm{HeLa}}{\mathrm{Ctrl} \text { PA }} \frac{\mathrm{BJ}}{\mathrm{Ctrl} \text { PA }} \frac{\mathrm{B} 16}{\mathrm{Ctrl} \text { PA }}$

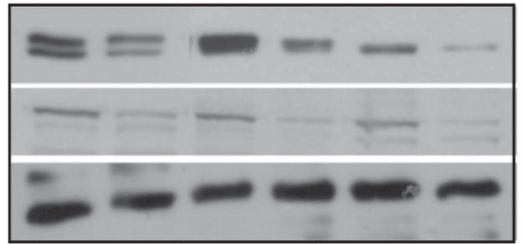

D

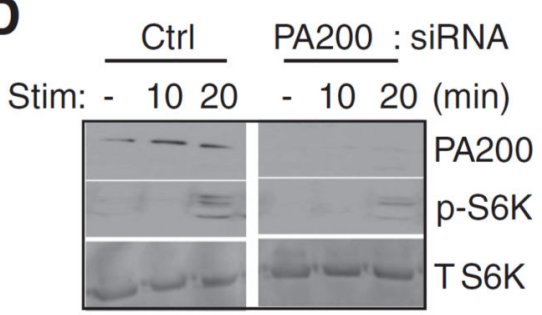

E

Ctrl PA200: siRNA

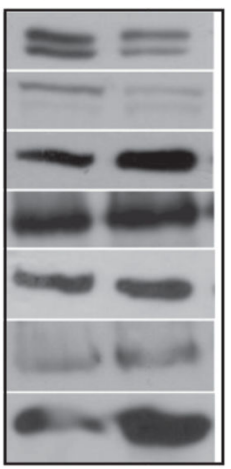

p-S6K

PA200

mTOR

Raptor

p-mTOR 2448

Rictor

p-mTOR 2481

F

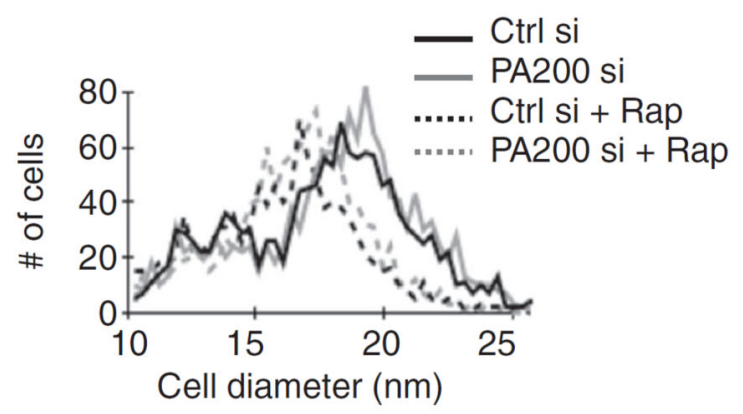

G
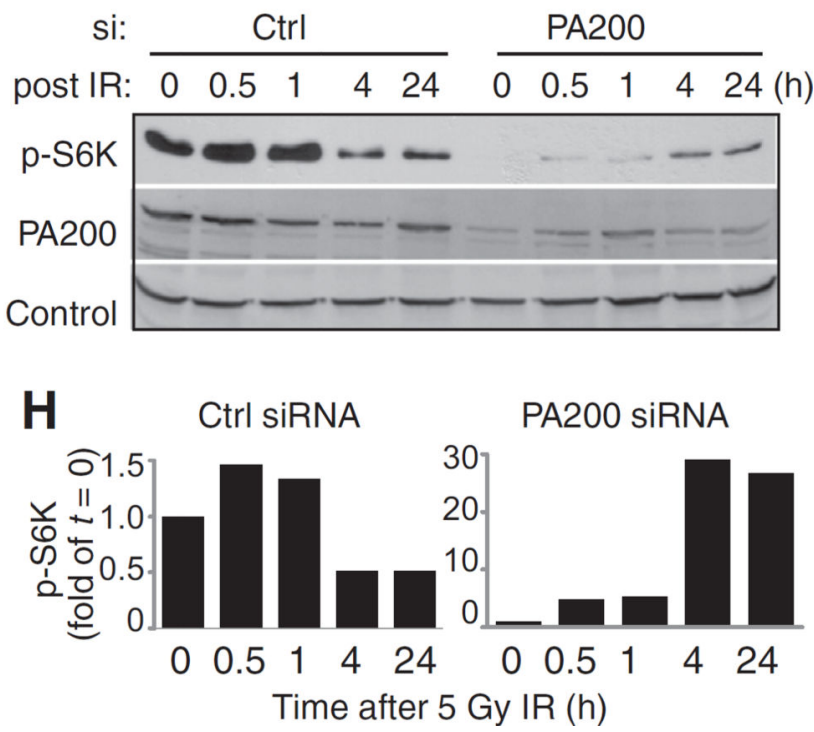

Figure 3.

PA200 is required for mTOR responsiveness. HeLa cells were transfected with PA200specific siRNA or nonspecific (Ctrl) siRNA and immediately plated $\left(14.4 \times 10^{4} \mathrm{cells} / \mathrm{cm}^{2}\right)$ in fresh media containing $2 \mathrm{mmol} / \mathrm{L}$ glutamine. Nutrients were replenished by daily replacement of media $\left(\right.$ Nutrient $\left.^{+}\right)$or cells were left in the same media $\left(\right.$Nutrient $\left.^{+}\right)$. Three days after transfection, cells were counted and viable cells were determined by Trypan blue exclusion (A) or harvested for Western blot analysis (B). Control (Ctrl) is HSP70. C, after transfection, the indicated cells were plated $\left(2 \times 10^{4} / \mathrm{cm}^{2}\right)$. Forty-eight hours after 
transfection, nutrients were replenished by media replacement. Twenty-four hours later, cells were harvested for Western blot analysis. Control is ERp57. D, after transfection, cells were plated at $2 \times 10^{4} / \mathrm{cm}^{2}$. Twenty-four hours after transfection, cell media were replaced with media lacking serum. Forty-eight hours after transfection, cells were stimulated with $10 \%$ bovine calf serum for the indicated times and then harvested for Western blot analysis. T, total. E, HeLa cells were treated as in (C) followed by Western blot analysis for the indicated proteins. $\mathrm{F}$, HeLa cells were treated as in (C) followed by cell diameter analysis by Vi-Cell counter (Becton Dickinson). Rap, rapamycin. G, HeLa cells transfected with PA200-specific siRNA or nonspecific siRNA (Ctrl) were plated and nutrients were replenished after 48 hours. Seventy-two hours after transfection, cells were harvested and replated. Replated cells were allowed to rest for 3 hours before irradiation. Cells were exposed to 5-Gy irradiation using Philips Mark 1 Cesium irradiator at a dose rate of $0.472 \mathrm{~Gy} / \mathrm{min}$. At the indicated times after radiation, cells were harvested for Western blot analysis of the indicated proteins. Control is HSP70. H, bands in (G) were quantified using ImageJ software. Signal of p-S6K band was normalized to the control for each time point and then the ratio was normalized to the ratio obtained at time zero. A representative figure of at least 3 independent experiments is shown for each panel. 
A

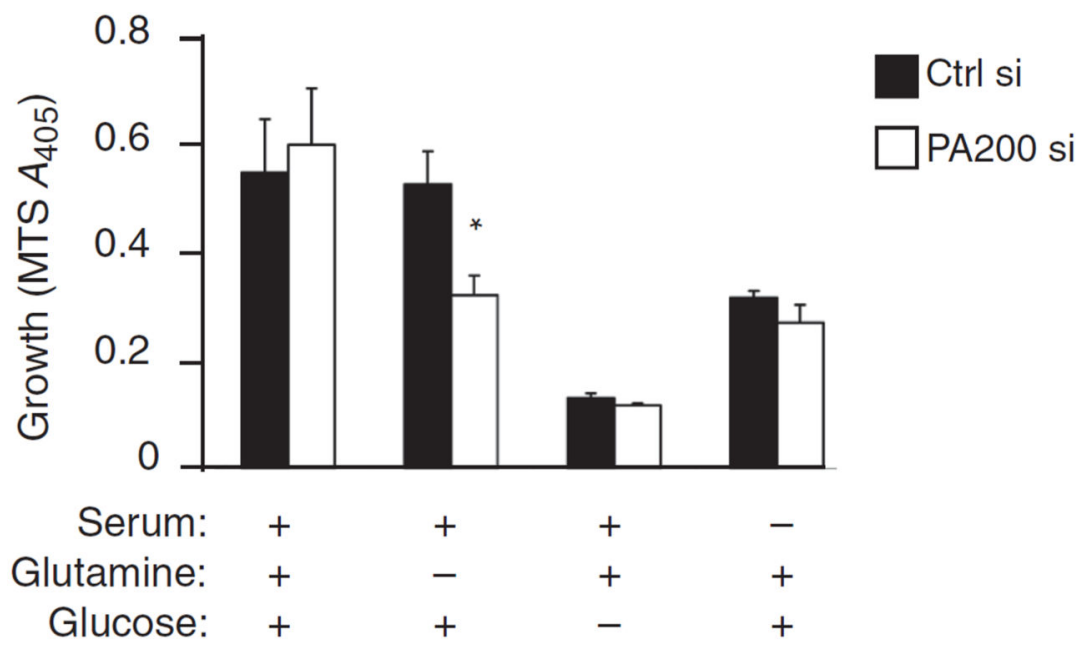

B
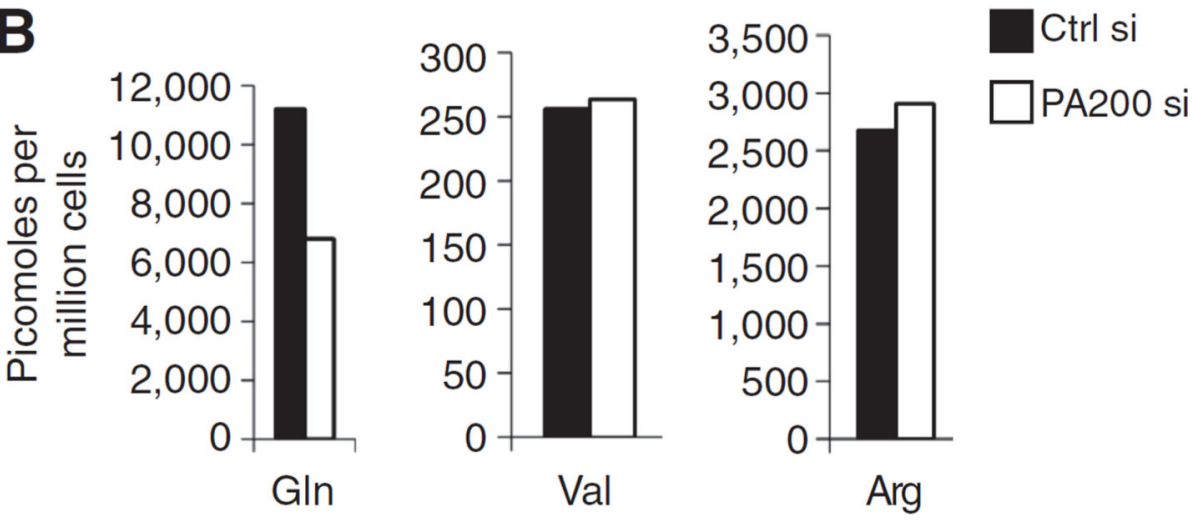

C

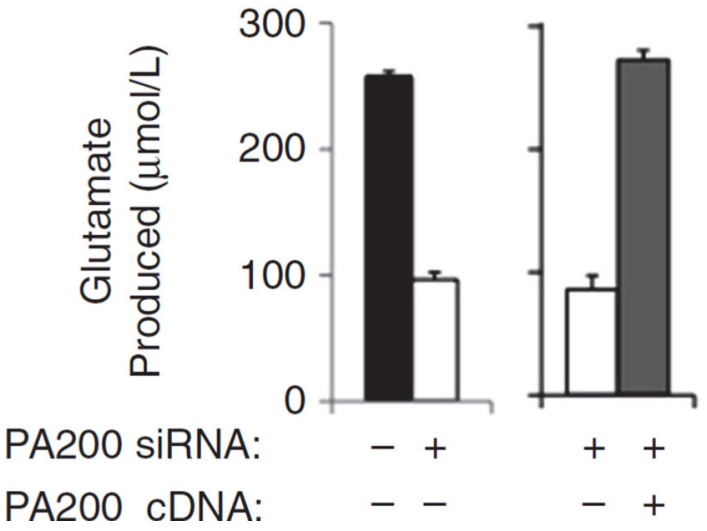

Figure 4.

PA200 is required to maintain intracellular free glutamine pools. A, after transfection, HeLa cells were immediately plated (10,000 cells per well of 96-well plate) in DMEM (which lacks glutamate) containing or lacking $10 \%$ bovine calf serum, $2 \mathrm{mmol} / \mathrm{L} \mathrm{L}$-glutamine, and $4.5 \mathrm{~g} / \mathrm{L}$ glucose. Five days later, cell growth/metabolic activity was measured using the Promega cell proliferation kit (MTS). B, HeLa cells were transfected with PA200-specific siRNA (white bars), control siRNA (black bars), or PA200-specific siRNA together with PA200 cDNA that is insensitive to the siRNA (gray bars). After media replacement on day 2 
after transfection, cells were allowed to grow for 3 more days. Deproteinized cell pellets (B) were analyzed for the indicated amino acids using the PicoTag method. Supernatants (C) were analyzed for glutamate remaining in the media using a BioProfile FLEX (Nova Biomedical). A representative figure of at least 3 independent experiments is shown for each panel. 